\title{
Innovative 2D nanomaterial integrated fiber optic sensors for biochemical applications
}

Xianfeng Chen, Miguel V. Andrés, Lin Zhang

Xianfeng Chen, Miguel V. Andrés, Lin Zhang, "Innovative 2D nanomaterial integrated fiber optic sensors for biochemical applications," Proc. SPIE 10681, Micro-Structured and Specialty Optical Fibres V, 1068107 (9 May 2018); doi: $10.1117 / 12.2307115$

SPIE. Event: SPIE Photonics Europe, 2018, Strasbourg, France 


\title{
Innovative 2D-nanomaterial integrated fiber optic sensors for biochemical applications
}

\author{
Xianfeng Chen ${ }^{\mathrm{a}, *}$, Miguel V. Andrés ${ }^{\mathrm{b}}$, Lin Zhang ${ }^{\mathrm{c}}$ \\ ${ }^{\text {a }}$ School of Electronic Engineering, Bangor University, Bangor LL57 1UT, United Kingdom \\ ${ }^{\mathrm{b}}$ Department of Applied Physics, University of Valencia, Valencia 46100, Spain \\ ${ }^{c}$ Aston Institute of Photonic Technologies, Aston University, Birmingham, United Kingdom
}

\begin{abstract}
We report our latest investigations on 2D-layered materials integrated fiber optic configurations for chemical and labelfree biosensing applications. Due to the favorable combination of exceptionally high surface-to-volume ratio and excellent optical and biochemical properties, graphene oxide (GO) and black phosphorus (BP) were deposited on fiber grating device as the bio-photonic linking layer to provide the remarkable platform for light-matter interface and affinity binding interaction. We developed a novel in-situ layer-by-layer (i-LbL) deposition technique based on chemicalbonding associated with physical-adsorption for the deposition of 2D materials. This approach secured high-quality 2D materials deposition on cylindrical fiber with strong adhesion as well as a prospective thickness control. By taking advantage of i-LbL deposition, the unique optical tunable features and polarization-selective characteristics have been experimentally observed. Several 2D material integrated fiber optic sensors have been proposed for chemical and biochemical applications, such as GO-long period grating (GO-LPG) based Hemoglobin sensor, GO dual-peak LPG (GO-dLPG) based label-free immunosensor, and the first BP fiber optic chemical sensor based on BP-tilted fiber grating (BP-TFG). We believe that 2D material integrated fiber optic configurations open the path as highly sensitive biophotonic platform for food safety, environmental monitoring, clinical diagnostics and biomedical applications.
\end{abstract}

Keywords: Graphene oxide; Black phosphorus; Fiber grating; Optical biosensor; Immunosensor; Hemoglobin; Heavy metal

\section{INTRODUCTION}

The development of biochemical sensor is extremely important for the healthcare, life science, clinical diagnosis, drug discovery, and environmental sectors ${ }^{1,2}$. To overcome the drawback of the traditional biosensor which is usually time consuming, complicated, labelling required and hazardous, fiber optic sensing technology has attracted great attention with the advantages of compact size, high sensitivity, label-free, real-time, multiplex and in-line determination ${ }^{3,4}$. Over past decades, fiber grating technology has been extensively developed by employing fiber Bragg gratings (FBGs), long period gratings (LPGs) and tilted fiber grating (TFGs) ${ }^{5-8}$. The major challenge is the lack of sensitivity for bioapplications with small biomolecules and low concentration of analyte.

To date, the advancement of 2D-layered materials plays an essential role in the exploration of multidisciplinary research in physics, chemistry, materials, medicine and biology ${ }^{9,10}$. Due to their exceptional properties of large surfaceto-volume ratio, atomic-thin layer structure and high carrier mobility, various 2D materials including graphene, graphene oxide (GO), transition metal dichalcogenides (TMDs), and black phosphorus (BP) have been developed for a wide range of applications in electronics, light processing, energy storages, chemical and biochemical sensors ${ }^{9-11}$. Moreover, GO displays advantageous characteristics for biosensing due to its excellent capabilities in biocompatibility, solubility and selectivity ${ }^{12}$. The enriched functional groups of GO can interact in an ionic, covalent or non-covalent manner, so that in principle they provide the highest extraction efficiency of biomolecules per unit area. The functionalized GO has been exploited to fabricate biosensors for drug delivery, bioimaging and cancer diagnostics. Recently, BP has emerged as a rising star in 2D material family ${ }^{13,14}$. Due to the unique puckered orthorhombic structure, BP has much large surface-tovolume ratio, extremely high hole mobility and superior molecular adsorption energy than those of other 2D materials.

*x.chen@bangor.ac.uk; phone (+ 44) 1248382480

Micro-Structured and Specialty Optical Fibres V, edited by Kyriacos Kalli, Alexis Mendez,

Christian-Alexander Bunge, Proc. of SPIE Vol. 10681, 1068107 • (c) 2018 SPIE

CCC code: $0277-786 X / 18 / \$ 18 \cdot$ doi: $10.1117 / 12.2307115$ 
The extraordinary properties of BP make it a very promising material for the applications in BP field-effect transistors (FETs), modulators, photo detectors, chemical sensors, and biosensing applications ${ }^{13-15}$.

In this paper, we introduce 2D material integrated fiber optic configurations as the bio-photonic platform for strong light-matter interface and for chemical and label-free biosensing applications. For nanomaterial-based sensors, one of the challenges is the limited sensitivity of monolayer. This can be overcome by integrating 2D-layered nanosheets with fiber gratings, which greatly enhances the light-matter interaction through the coupling between evanescent wave and nanocoating. A novel in-situ layer-by-layer (i-LbL) deposition technique based on chemical-bonding associated with physical-adsorption has been developed to deposit 2D-layered nanosheets on non-planar substrate (e.g. cylindrical fiber). This approach could secure high-quality nano-coating on specific cylindrical fiber surface with strong adhesion as well as a prospective thickness control. The surface morphology was characterized by atomic force microscopy (AFM), scanning electron microscope (SEM), and Raman spectroscopy. Due to its excellent capabilities, GO provides a remarkable analytical platform for bio-affinity binding interaction. The GO-LPGs were proposed for Hemoglobin detection and label-free IgG/Anti-IgG immunosensing. The BP-TFG was exploited as the first BP-fiber optic chemical sensor for heavy metal $\left(\mathrm{Pb}^{2+}\right.$ ions) detection, showing the ultrahigh sensitivity, much lower limit of detection (LOD) and extremely wide concentration sensing range. The proposed nanomaterial-fiber optic configurations could open the path as bio-photonic platform for food safety, environmental monitoring, clinical diagnostics and biomedical applications.

\section{GRATING FABRICATION, PRINCIPLE, DEPOSITION, SENSITIVITY}

\subsection{Fabrication of fiber gratings}

In this work, three types of gratings were fabricated: 1) a $30 \mathrm{~mm}$-long dual-peak LPG (dLPG) with period of $162 \mu \mathrm{m}$, 2) a $15 \mathrm{~mm}$-long LPG with period of $400 \mu \mathrm{m}$, and 3) a $12 \mathrm{~mm}$-long TFG with tilted angle of $81^{\circ}$. All the gratings were UV-inscribed in hydrogen loaded SMF fiber by using frequency-doubled Ar laser and multiple scanning technique. After UV fabrication, the grating were annealed at $80^{\circ} \mathrm{C}$ for $48 \mathrm{~h}$ to remove the residual hydrogen and to stabilize the optical properties.

\subsection{Principle of LPG and TFG}

A long period grating is typically formed by a periodic refractive index (RI) modulation in the order of hundreds of micrometers in fiber core. The perturbation of RI in fiber core results in light coupling from the fundamental core mode to the forward-propagating cladding modes, yielding a series of attenuation bands in transmission spectrum with corresponding wavelength satisfying the phase-matching condition ${ }^{16}$ :

$$
\lambda_{\text {res }}=\left(n_{c o}^{e f f}-n_{c l, i}^{e f f}\right) \Lambda
$$

where $n_{c o}^{e f f}$ and $n_{c l, i}^{e f f}$ are the effective refractive indices of core and $i$ th cladding mode, $\Lambda$ is the grating period. The transmission power $T$ of the attenuation bands is given by:

$$
T=1-\sin ^{2}(\kappa \mathrm{L})
$$

where $L$ is the length of grating, and $\kappa$ is the coupling coefficient between $L P_{v j}$ and $L P_{\mu k}$ mode:

$$
\kappa=\frac{\omega}{4 P_{0}} \int_{\phi=0}^{2 \pi} \int_{r=0}^{\infty} \Delta \varepsilon(r, \phi, z) \psi_{v j}(r, \phi) \psi_{\mu k}^{*}(r, \phi) r d r d \phi
$$

where $\omega$ is the FWHM of grating profile, $P_{0}$ is the power of the mode, $\Delta \varepsilon(r, \varphi, z)$ is the permittivity variation, $\psi(r, \phi)$ is the transverse field of the cladding mode, and $r$ and $\phi$ represent radial and angular field, respectively.

The coating layer could make a transition from cladding guided modes to overlay guided modes as well as radiation modes ${ }^{17,18}$. For a conventional bare LPG, the highest RI sensitivity occurs at the surrounding-medium RI (SRI) value approaching the cladding RI (CRI) by the higher order mode. When the SRI exceeds the CRI, the core mode couples with radiation modes and the phase-matching will no longer be satisfied. For thin-film coated LPG, both resonant wavelength and intensity show unique properties against the SRI. The coupling coefficient is determined by the overlap integral of core and cladding modes and by the amplitude of the periodic modulation of the mode propagation constants. For a bare LPG, the intensity $T$ changes slowly with the increase of SRI, providing poor sensitivity as intensity sensor. For a thin-film coated-LPG, the coupling strength would change and enable as intensity sensor ${ }^{19-21}$.

For the TFG with largely titled angle (e.g. $\theta=81^{\circ}$ ), the light is coupled from core to co-propagating cladding modes at the wavelength determined by the phase-matching condition $^{16}$ : 


$$
\lambda_{c o-c l}=\left(n_{c o, i}^{e f f}-n_{c l, m}^{e f f}\right) \cdot \frac{\Lambda_{g}}{\cos \theta}
$$

where $n_{c o, i}^{e f f}$ is the effective index of fiber core, $i$ represents transverse-magnetic (TM) or transverse-electric (TE) polarization state, $n_{c l, m}^{e f f}$ is the effective index of cladding, $m$ means $m$ th mode, $\Lambda_{g}$ is the normal period of grating, and $\theta$ is the tilted angle.

\subsection{Deposition of 2D-layered nanosheets on fiber surface}

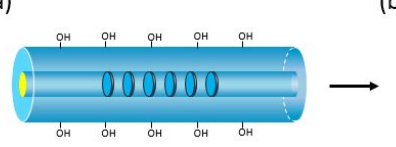

(d)
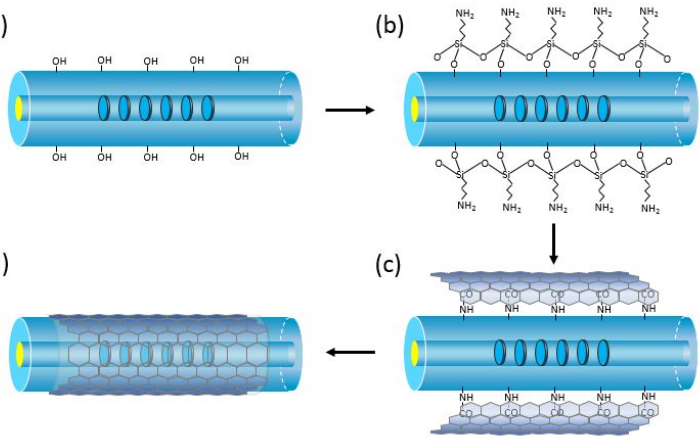

Fig. 1. Schematic of 2D nanosheets deposition on fiber.

We develop a novel i-LbL technique for 2D-layered nanosheets deposition on cylindrical fiber substrate, which is based on chemical-bonding associated with physicaladsorption. As shown in Fig. 1, the fiber sample was initially cleaned with acetone to remove the residual contaminan, then it was treated by immersion in $1.0 \mathrm{M} \mathrm{NaOH}$ solution for $1 \mathrm{~h}$ at room temperature to enrich the number of hydroxyl group $(-\mathrm{OH})$ on fiber surface, washed with deionized (DI) water thoroughly and dried (Fig. 1a). For the silanization (Fig. 1b), the alkaline-treated fiber was firstly immersed in 5\% APTES solution for $1 \mathrm{~h}$ at room temperature to form $\mathrm{Si}-\mathrm{O}-\mathrm{Si}$ bonding on the surface, followed by washing with ethanol to remove unbound monomers and baked in an oven at $70^{\circ} \mathrm{C}$ for $30 \mathrm{~min}$ to improve the stability of APTES monolayer. After silanization, the fiber was incubated in 2D nanosheets dispersion contained in a custom-made mini-bath, which was placed on a hot plate at $42{ }^{\circ} \mathrm{C}$. The epoxy group of $2 \mathrm{D}$ materials reacted with amino group of APTES-silanized fiber, hence nanosheets were gradually bonded on fiber surface (Fig. 1c) while the solvent was being evaporated. Afterwards, the followed multiple cycles of physical-adsorption procedures were conducted, where solid 2D nanosheets were gradually and physically adsorbed onto fiber surface.

\subsection{Surface morphological characterization}

The surface morphology of 2D nanosheets-coated fiber was characterized by optical microscope, SEM, AFM and Raman spectroscopy. The initial microscope image (Fig. 2a) shows a clear boundary between bare and GO-coated sections, demonstrating a successful deposition. The surface coverage was examined by SEM with the magnification of $1000 \times$. The detailed texture in Fig. $2 b$ indicates that the GO nanosheets have been deposited onto fiber to form a homogeneous layer over the entire cylindrical surface. Moreover, the thickness of GO overlay was determined by AFM. As shown in AFM image and the height profile (Fig. 2c), the thickness of GO overlay is identified as around $501.8 \mathrm{~nm}$ for GO-LPG device. As shown in Fig. 2d, there are three characteristic peaks at 1335,1599 , and $2682 \mathrm{~cm}^{-1}$ for GO-coated fiber, indicating the success of $\mathrm{GO}$ deposition.

Please note that overlay thickness is only $49.2 \mathrm{~nm}$ for GO-dLPG and $323 \mathrm{~nm}$ for BP-TFG in following work.
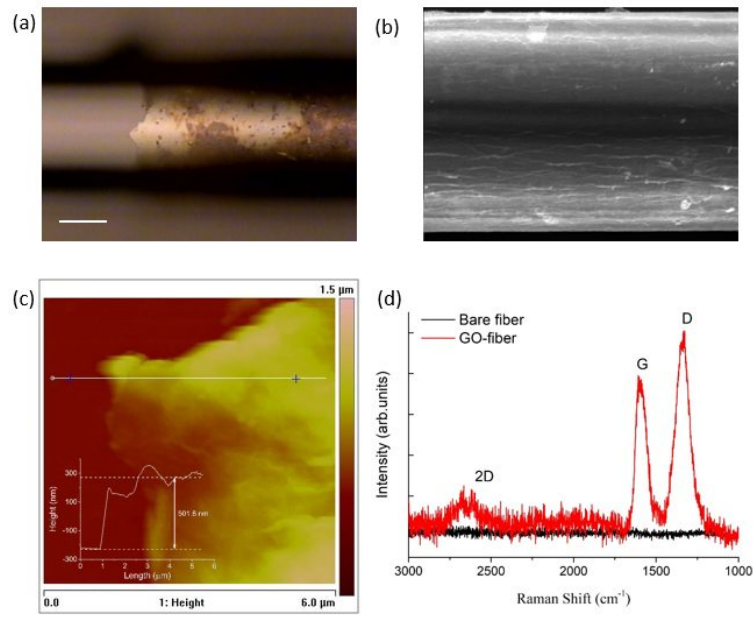

Fig. 2. Surface morphology of GO-fiber. (a) Optical microscope image (bar: $50 \mu \mathrm{m}$ ), (b) SEM image, (c) AFM image (inset: height profile), and (d) Raman spectra.

\subsection{Enhanced sensitivity of GO-coated LPG}

The GO-coated LPG was implemented for RI sensing with a set of RI solutions with the RI values from 1.3315 to 1.4615. In order to avoid the bend effect, the GO-LPG was placed in a straight V-groove with two ends fixed and the solution was added into $\mathrm{V}$-groove by careful pipetting to cover the entire grating region. After each measurement, both grating device and V-groove were rinsed with methanol thoroughly. Fig. 3a plots the transmission spectra of GO-LPG against different RIs. There are two different trends for RI region below and above CRI $(\approx 1.445)$. For SRI lower than CRI, the resonant intensity decreases by increasing SRI, which is due to the reduction of coupling coefficient Eq.(3). 
Once SRI is equal to CRI, the cladding modes are no longer confined by the cladding layer which is acting as an infinite medium and supports no discrete cladding modes. When SRI is higher than CRI, the fiber does not support any bound cladding mode and the re-appeared resonance corresponds to leaky mode coupling. By further increasing SRI, the resonant intensity increases because the leaky mode is better confined by the Fresnel reflection while its wavelength is influenced with a blue-shift.

The resonant intensity change against SRI is plotted in Fig. 5b. For RI region below CRI, the intensity shows a nonlinear behavior for a gradual decrease by increasing RI which is consistent with those LPGs coated with CNT, ZnO, $\mathrm{TiO}_{2}{ }^{19-21}$. The sensitivity in intensity achieves $-76.5 \mathrm{~dB} / \mathrm{RIU}$ and $-234.2 \mathrm{~dB} / \mathrm{RIU}$ for RI range of 1.33-1.38 and 1.40-1.44, respectively, exhibiting 2.5 times and 5 times higher than that of CNT-deposited LPG for the corresponding RI ranges. For RI higher than CRI, the resonant intensity increases dramatically against RI. The sensitivity approaches +1580.5 $\mathrm{dB} / \mathrm{RIU}$ which is 7.3 times higher than that of ZnO-coated LPG. For the case of RI=1.4615, the resonant intensity reaches $-28.35 \mathrm{~dB}$, indicating that around $99.8 \%$ of core mode has been coupled into leaky mode, which is extremely high than that of metal oxide-coated LPGs. The enhanced sensitivity of GO-LPG may be caused by the unique features of GO material, such as extraordinary large surface-to-volume ratio, high carrier mobility, and excellent optical properties. GO provides strong light-matter interaction between evanescent field and surrounding-medium.
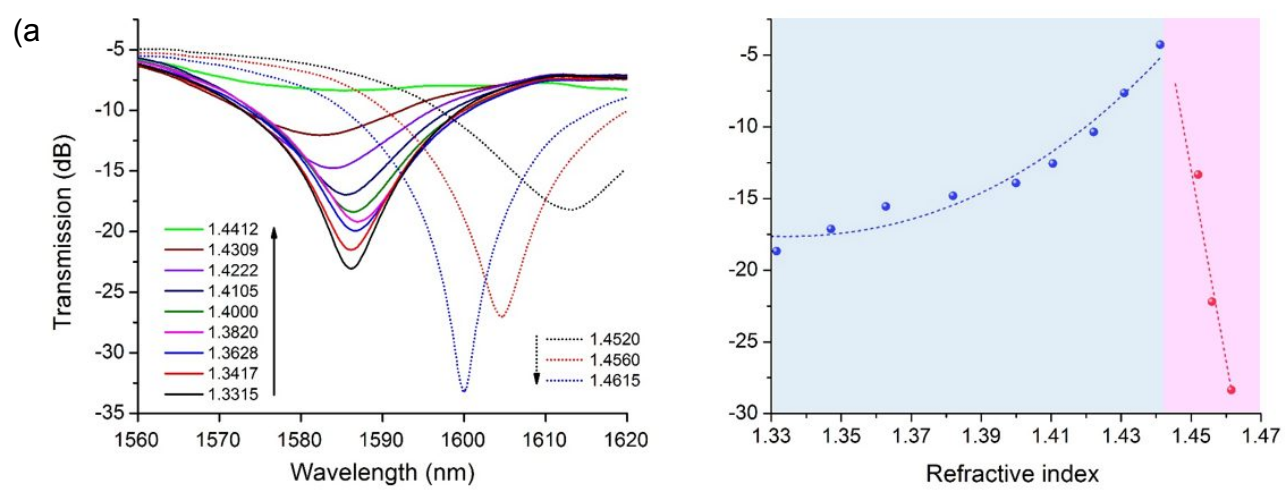

Fig. 3 GO-LPG (a) transmission spectra and (b) resonant intensity change against RI.

\section{CHEMICAL AND BIO- SENSING APPLICATIONS}

\subsection{Detection of human hemoglobin}

The GO-LPG was performed as a biosensor to detect human hemoglobin. A set of hemoglobin concentrations ranging from 0.0, $0.2,0.4,0.6,0.8$ and $1.0 \mathrm{mg} / \mathrm{mL}$ were prepared with sucrose solution $(\mathrm{RI}=1.4610)$ acting as RI buffer. As shown in Fig. 4a, the resonant intensity increases by $1.91 \mathrm{~dB}$ when the hemoglobin concentration changes from 0.0 to $1.0 \mathrm{mg} / \mathrm{mL}$. Defining the concentration sensitivity as the change induced by $1 \mathrm{mg} / \mathrm{mL}$ hemoglobin, we have the device sensitivity of $1.9 \mathrm{~dB} /(\mathrm{mg} / \mathrm{mL})$. If use a low-noise interrogation system with a resolution of $0.1 \mathrm{~dB}$, the GO-LPG could detect a hemoglobin concentration change as small as $0.05 \mathrm{mg} / \mathrm{mL}$, which is far below the hemoglobin threshold value for anemia (130 mg/mL for man and $120 \mathrm{mg} / \mathrm{mL}$ for women) defined by $\mathrm{WHO}^{22}$.
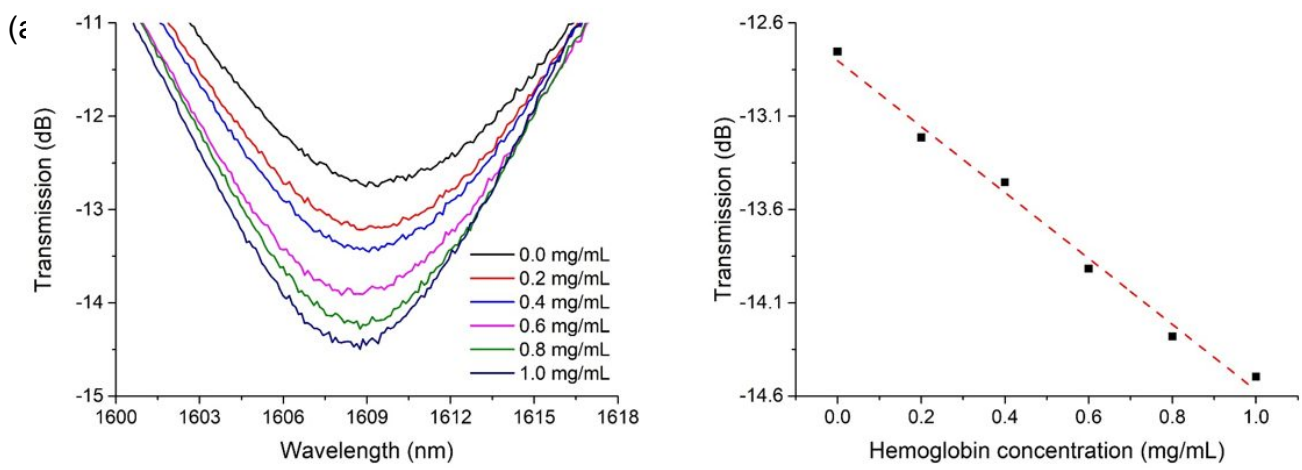

Fig. 4 (a) Transmission spectra and (b) resonant intensity change of GO-LPG against hemoglobin concentrations. 
The increase of resonant intensity could attribute to the local RI change caused by the adsorption of hemoglobin molecules onto $\mathrm{GO}$, hence the strongest $\pi-\pi$ interactions lead to the most effective adsorption of proteins onto GO. GO provides a significant sensing linkage between evanescent field and target biomolecules with enhanced light-matter interaction, consequently exhibiting ultrahigh sensitivity for hemoglobin detection.

\subsection{GO-dLPG for label-free immunosensing}

Biofunctionalization of GO-dLPG: The immobilization of probe biomolecules on device surface plays a crucial role in generating a biosensor. The GO-dLPG with $49.2 \mathrm{~nm}$-thick overlay was developed for immunosensor.
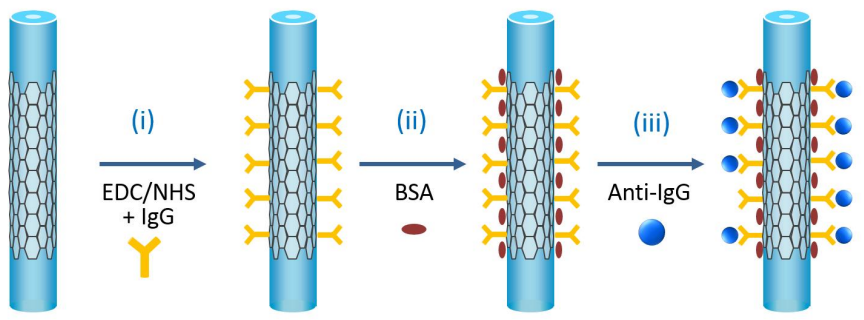

Fig. 5 illustrates the functionalization of a GOdLPG based biosensor. (i) The GO-dLPG was immersed into a mixture of EDC/NHS solution for $1 \mathrm{~h}$. Then it was sunk into a rabbit IgG with a concentration of $1 \mathrm{mg} / \mathrm{mL}$ for $2 \mathrm{~h}$ to immobilize the probe IgG on GO surface. GO reacted with EDC to yield a stable active ester in the presence of NHS while the ester reacted with the amine group of $\operatorname{IgG}$ to form a covalent immobilization leaving the binding sites free for recognition of anti-IgG. (ii) The unreacted sites on GO surface were passivated by bovine serum albumin (BSA),

Fig. 5 Schematic of functionalization of GO-dLPG based biosensor.

the IgG-bound GO-dLPG was immersed into $1 \%$ BSA solution for 30 min to block the remaining activated carboxylic group and to prevent non-specific adsorption onto GO surface. (iii) Here, the IgG-bound GO-dLPG was ready as a biosensor for the detection of target anti-IgG.

Label-free immunosensing of antibody-antigen kinetic interaction: The fiber device was placed straight in a custommade V-groove container on a Teflon plate to minimize the bend cross-sensitivity. All the chemicals and solvents were added and withdrawn by careful pipetting. The resonance $L P_{012}^{\text {red }}$ of GO-dLPG was selected to monitor the spectral evolution throughout the biosensing process. Four consecutive processes for different goat anti-rabbit IgG concentrations of $1,10,50$, and $100 \mu \mathrm{g} / \mathrm{mL}$ were plotted in Fig. 6. Each process was performed in three stages: i) Pre-wash stage, the rabbit IgG-bound GO-dLPG was washed by PBS buffer (1×PBS, pH 7.4) for 5 min, providing a stable baseline. ii) Kinetic binding stage when the IgG-bound sensor was immersed in goat anti-rabbit IgG solution. The first 3 min was a rapid reaction process during which the resonance dramatically moved to long wavelength, followed by a 27 min steady process when the signal was gradually moving and finally achieving the saturation. iii) A subsequent rinsing with PBS buffer thoroughly to remove unbound anti-IgG prior to the next measurement. During four antibody-antigen binding processes, the grating peak wavelength shifts as absolute change in RI after deducting the baseline signal in PBS were $1470,1730,2415$, and $1960 \mathrm{pm}$ for anti-IgG concentrations of $1,10,50$, and $100 \mu \mathrm{g} / \mathrm{mL}$, respectively. The total wavelength shift corresponding to the whole binding was $4735 \mathrm{pm}$.
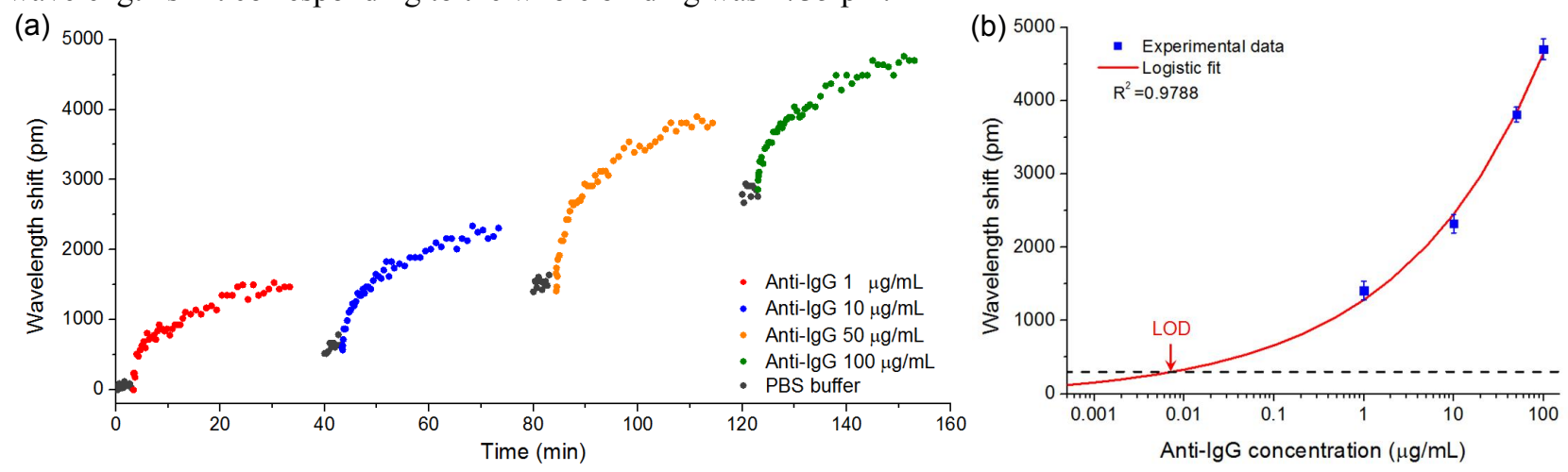

Fig. 6 (a) Wavelength shift against time during IgG and Anti-IgG binding interaction processes. (b) Dependence of wavelength shift against anti-IgG concentrations.

The wavelength shift as a function of the concentration of anti-IgG has been plotted in Fig. 6b. The red line provides the best logistic curve fitting of the experimental data. The LOD is estimated below $7 \mathrm{ng} / \mathrm{mL}$, which is 100 -fold better 
than LPG-based immunosensor ${ }^{23}$. The mechanism of biosensing is that the bioaffinity binding changes local analyte concentration, increasing the local RI at the GO-analyte interface, where the evanescent wave penetrates, hence induces an optical signal change.

\subsection{BP-TFG based chemical sensor for heavy metal detection}

$B P$ nanosheets synthesis and deposition: BP nanosheets were synthesized by using a liquid-phase exfoliation method where the absolute ethanol was used as solvent. The BP nanosheets were deposited on TFG surface by the developed i-LbL technique. Afterwards, the surface morphological characterization was investigated by Raman spectroscopy, SEM and AFM. The thickness of BP overlay was $323 \mathrm{~nm}$.

Optical tunable properties: For a $81^{\circ}-\mathrm{TFG}$, the excessively tilted plane of grating breaks the symmetry of mode coupling, inducing the significant polarization dependent coupling characteristics. $81^{\circ}$-TFG exhibits two sets of comb-like split resonances in transmission spectra, which can be fully excited by launching the linearly polarized light. Spectra (i) in Fig. 7a show a zoomedpair of polarization modes, in which TM and TE modes have been fully excited, respectively. The transmission spectra of BP-TFG were monitored during BP deposition process and recorded as (ii), (iii),
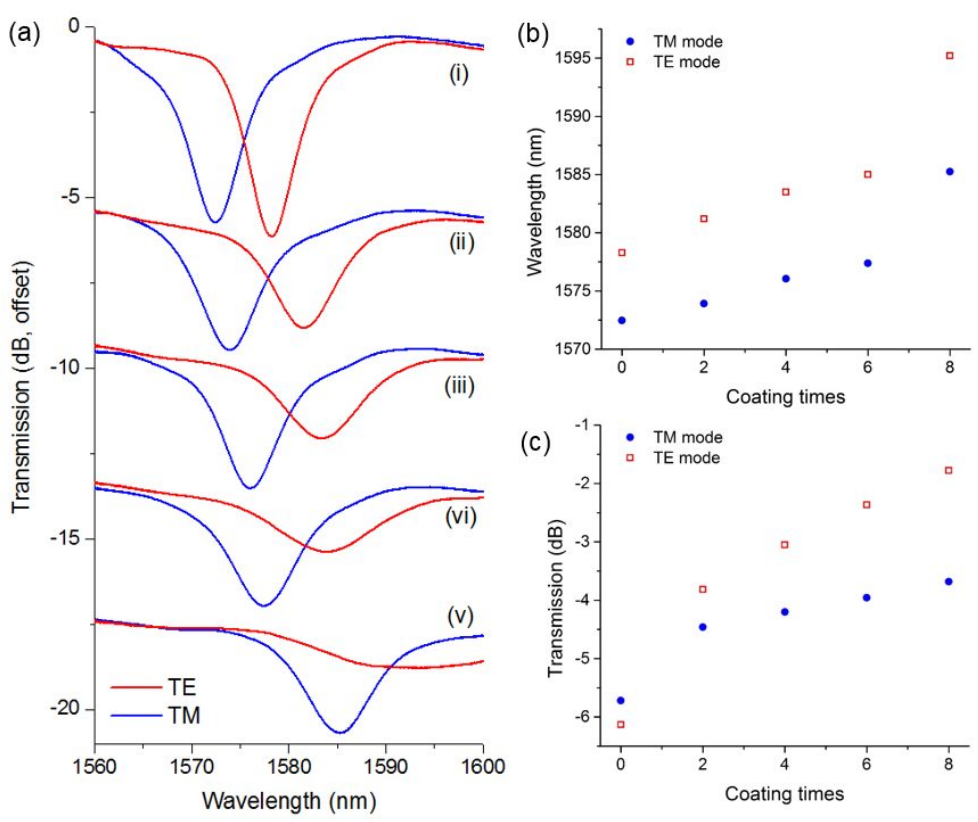

Fig. 7 (a) Spectral evolution during BP-deposition process revealing strong light-matter interface and optical tunable features. (b) Wavelength shift and (c) intensity change against BP deposition cycles. (iv) and (v) in Fig. 7 (a) after $2^{\text {nd }}, 4^{\text {th }}, 6^{\text {th }}$ and $8^{\text {th }}$ coating cycle, respectively.

The properties of thickness-dependent polarization and polarization-selective coupling were experimentally observed. Both TM- and TE -polarized resonances show a significant red-shift in wavelength and a decrease in intensity against the increase of BP overlay thickness. The wavelength shift was attributed to the increase of cladding effective RI induced by high RI coating layer, and consequently the phase-matching condition Eq. (4) was tuned. The leaky radiation from cladding to coating layer could reduce the coupling coefficient between core and cladding modes hence decrease the resonant intensity. However, the variation of TE mode is always larger than that of TM mode. After 8 cycles of deposition, TE mode has $16.9 \mathrm{~nm}$ in wavelength shift (Fig. 7b) and $4.35 \mathrm{~dB}$ in intensity decrease (Fig. 7c), which are 1.2 times and 2.1 times larger than those of TM mode, respectively. The separation between two polarized resonances of BPTFG has been increased to $8.38 \mathrm{~nm}$, giving an estimated birefringence of $\sim 3.0 \times 10^{-4}$ which is $150 \%$ enhancement that of bare $81^{\circ}$-TFG.

Detection of heavy metal $\mathrm{Pb}^{2+}$ : The BP-TFG was exploited for the implementation of BP strong ability of lead ions adsorption and ultrahigh sensitivity for heavy metal chemical sensing. A series of aqueous solutions containing $\mathrm{Pb}^{2+}$ ions with concentrations of $0.1,1.0,10,100,1000,1 \times 10^{4}, 1 \times 10^{5}, 1 \times 10^{6}$ and $1.5 \times 10^{7} \mathrm{ppb}$ were prepared for the detection. The BP-TFG was firstly immersed into $\mathrm{Pb}^{2+}$ solution for $120 \mathrm{~s}$, then the solution was withdrawn by careful pipetting to expose the BP-TFG in the air for $180 \mathrm{~s}$ when the transmission spectrum achieved steady and was captured by OSA. Afterwards, the BP-TFG was washed with ethanol thoroughly to remove the adsorbed lead ions prior to next concentration measurement. As plotted in Fig. 8a, the peak shows apparent intensity decrease by increasing $\mathrm{Pb}^{2+}$ concentration, indicating the strong absorption occurred between BP and lead ions. Fig. $8 \mathrm{~b}$ depicts the intensity change as a function of ion concentrations, revealing a non-linear relationship with piecewise sensitivities of $0.5 \times 10^{-3}, 7.7 \times 10^{-7}$ and $2.3 \times 10^{-8} \mathrm{~dB} / \mathrm{ppb}$ for $\mathrm{Pb}^{2+}$ concentration ranges of $0 \sim 100 \mathrm{ppb}, 10^{3} \sim 10^{5} \mathrm{ppb}$, and $10^{6} \sim 10^{7} \mathrm{ppb}$, respectively. The proposed $\mathrm{BP}-\mathrm{TFG}$ can effectively detect $\mathrm{Pb}^{2+}$ ions concentration over a wide range from $0.1 \mathrm{ppb}$ to $1.5 \times 10^{7} \mathrm{ppb}$ with no 
saturation effect, which is four orders of magnitude larger than that of BP-FET based lead sensor ${ }^{24}$. By the best logistic fitting of the experimental data (Fig. 8b), the LOD was estimated below $0.25 \mathrm{ppb}$ for $\mathrm{Pb}^{2+}$ ions, which is 4 -fold better than CNTs functionalized heavy metal sensor ${ }^{25}$. Meanwhile, this LOD of $0.25 \mathrm{ppb}$ is only one fortieth of WHO's permissible limit of $10 \mathrm{ppb}$ for lead in drinking-water ${ }^{26}$. For comparison, the similar detection was also conducted for light metal $\mathrm{Na}^{+}$ions with the results plotted as green symbols in Fig. 8b, showing a very limited sensitivity. It reveals that BP exhibits strong adsorption of heavy metal ions hence the ultrahigh sensitivity for lead ions detection. When the carrier concentration was large enough, correlation effects became significant and resulted in an increase of carrierinduced RI change. Consequently, the fiber guided wave was modulated by perturbing phase condition and optical absorption coefficient.
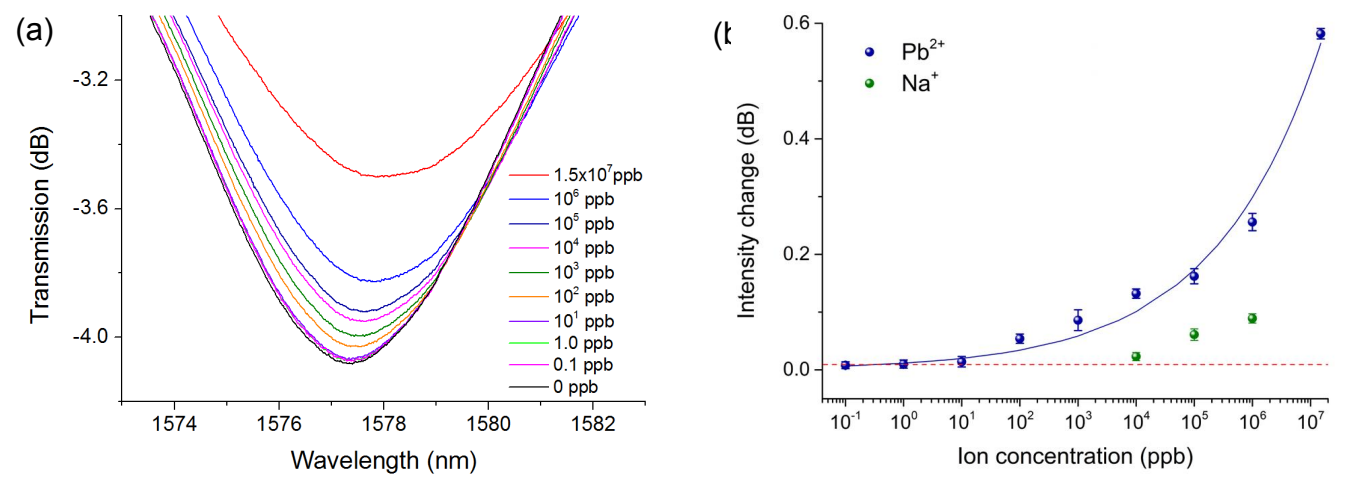

Fig. 8 TM-peak (a) transmission spectra and (b) intensity changes against $\mathrm{Pb}^{2+}$ (blue) and $\mathrm{Na}^{+}$(blue) concentrations.

\section{CONCLUSION}

We proposed 2D-layered materials integrated fiber optic configurations. A novel i-LbL deposition technique was developed with the advantages of the secured high-quality 2D materials deposition on cylindrical fiber with strong adhesion as well as a prospective thickness control. The unique optical tunable features and polarization-selective characteristics were experimentally observed. The proposed sensors have been implemented for the detections of hemoglobin, IgG/anti-IgG interaction, and heavy metal ions, demonstrating ultrahigh sensitivity, lower LOD and significant sensing performance. The novel 2D material-fiber optic configuration open the path for superior chemical and biomedical applications.

\section{ACKNOWLEDGEMENTS}

The authors acknowledge the support from the EU FP7 PIRSES-2013-612267 (iPhoto-Bio) and Sêr Cymru NRN Fund (NRN097), and the contributions from Mr C Liu and Mr Q Cai for this work.

\section{REFERENCES}

[1] R.S. Marks, C. R. Lowe, D. C. Cullen, H. H. Weetall, I. Karube, Handbook of Biosensors and Biochips. Wiley. 2007.

[2] M. Estevez, M. A. Otte, B. Sepulveda, L. M. Lechuga, Analytica Chimica Acta 2014. 806, 55-73.

[3] X. D. Wang, O. S. Wolfbeis, O.S., Anal Chem. 2013, 85, 487-508.

[4] A. Cusano, M. Consales, A. Crescitelli, A. Ricciardi, (Eds.) Springer. 2014.

[5] S. W. James and R. P. Tatam, Meas. Sci. Technol. 2003, 14, R49-R61.

[6] X. Chen, L. Zhang, K. Zhou, E. Davies, K. Sugden, I. Bennion, M. Hughes, A. Hine, Opt. Lett. 2017, 32, $2541-2543$.

[7] K. Zhou, L. Zhang, X. Chen, and I. Bennion, J. Lightw. Technol. 2006, 24, 5087.

[8] J. Albert, L. Y. Shao, C. Caucheteur, Laser and Photonics Reviews 2013, 7(1), 83-108.

[9] K. S. Novoselov, A. K. Geim, S. V. Morozov, D. Jiang, Y. Zhang, S. Dubonos, I. Grigorieva, A. Firsov, Science 2004, $306,666$.

[10] A. K. Geim, Science 2009, 324, 1530.

[11] Z. Sun, A. Martinez, F. Wang, Nature Photon. 2016, 10, 227-238.

[12] K. P. Loh, Q. Bao, G. Eda, M. Chhowalla, Nat. Chem. 2010, 2, 1015-1024.

[13] L. Li, Y. Yu, G. J. Ye, Q. Ge, X. Ou, H. Wu, D. Feng, X. H. Chen, Y. Zhang, Nat. Nanotechnol. 2014, 9, $372-377$. 
[14] F. Xia, H. Wang, and Y. Jia, Nat. Commun. 2014, 5, 4458.

[15] A. Castellanos-Gomez, J. Phys. Chem. Lett. 2015, 6, 4280-4291.

[16] T. Erdogan, J. Opt. Soc. Am. A, 1997, 14(8) 1760-1773.

[17] I. Del Villar, I. R. Matías, F. J. Arregui, P. Lalanne, Opt. Express 2015, 13(1), 56-69.

[18] A. Cusano, A. Iadicicco, P. Pilla, L. Contessa, S. Campopiano, A. Cutolo, M. Giordano, Opt. Express 2006, 14(1), $19-34$.

[19] Y. C. Tan, W. B. Ji, V. Mamidala, K. K. Chow, and S.C. Tjin, Sens. Actuators B 2014, 196, 260-264.

[20] L. Coelho, D. Viegas, J.L.Santos, and J.M.M.M. de Almeida, Sens. Actuators B 2016, 223, 45-51.

[21] L. Coelho, D.Viegas, J.L. Santos, and J.M.M.M. de Almeida, Sens. Actuators B 2014, 202, 929-934.

[22] World Health Organization. Nutritional anemias: report of a WHO scientific group. Switzerland, 1968.

[23] DeLisa, M.P., Zhang, Z., Shiloach, M., Pilevar, S., Davis, C.C., Sirkis, J.S., Bentley, W.E., Anal. Chem. 2000. 72, $2895-2900$.

[24] P. Li, D. Zhang, J. Liu, H. Chang, Y. Sun, and N. Yin, ACS Appl. Mater. Interfaces 2015, 7, 24396-24402.

[25] M. Shaban and A. R. Galaly, Sci. Rep. 2016, 6, 25307.

[26] World Health Organization. Guidelines for Drinking-water Quality, 4th ed. 2011. 